\title{
The nonlinear redshift-space power spectrum of galaxies
}

\author{
A.F. Heavens ${ }^{1}$, S. Matarrese ${ }^{2}$, L. Verde ${ }^{1}$ \\ 1 Institute for Astronomy, University of Edinburgh, Royal Observatory, Blackford Hill, Edinburgh EH9 3HJ , United Kingdom \\ 2 Dipartimento di Fisica Galileo Galilei, Università di Padova, via Marzolo 8, I-35131 Padova, Italy
}

1 February 2008

\begin{abstract}
We study the power spectrum of galaxies in redshift space, with third order perturbation theory to include corrections that are absent in linear theory. We assume a local bias for the galaxies: i.e. the galaxy density is sampled from some local function of the underlying mass distribution. We find that the effect of the nonlinear bias in real space is to introduce two new features: first, there is a contribution to the power which is constant with wavenumber, whose nature we reveal as essentially a shot-noise term. In principle this contribution can mask the primordial power spectrum, and could limit the accuracy with which the latter might be measured on very large scales. Secondly, the effect of second- and third-order bias is to modify the effective bias (defined as the square root of the ratio of galaxy power spectrum to matter power spectrum). The effective bias is almost scale-independent over a wide range of scales. These general conclusions also hold in redshift space. In addition, we have investigated the distortion of the power spectrum by peculiar velocities, which may be used to constrain the density of the Universe. We look at the quadrupole-to-monopole ratio, and find that higher-order terms can mimic linear theory bias, but the bias implied is neither the linear bias, nor the effective bias referred to above. We test the theory with biased N-body simulations, and find excellent agreement in both real and redshift space, providing the local biasing is applied on a scale whose fractional r.m.s. density fluctuations are $<0.5$.
\end{abstract}

Key words: cosmology: theory - galaxies: clustering and redshift - galaxies: bias large-scale structure of Universe

\section{INTRODUCTION}

The clustering properties of galaxies are dependent to a certain extent on the precise population analysed. This implies that not all populations can be unbiased tracers of the matter distribution - at least one must be biased. Simple bias models have been in existence since the early Cold Dark Matter (CDM) simulations suggested that pairwise velocities of galaxies would be too high unless the (optically-selected) galaxies were more strongly clustered than the mass (Davis et al. 1985). In the first simple models, it was assumed that the fractional overdensity in galaxies $\delta^{g} \equiv \delta n / n$ was simply a multiple of the mass overdensity: $\delta^{g}=b \delta$. This linear bias model cannot be true in detail for all populations, since the shape of the power spectrum is unchanged in this case, and not all galaxy populations have the same shape of power spectrum, although the differences are not large (Peacock \& Dodds 1994). At a more fundamental level, such a model could only survive if applied to a smoothed field, otherwise $\delta^{g}<-1$ which corresponds to a negative galaxy density. The linear bias model can be viewed as an approximation to a more general Eulerian bias prescription, where the galaxy density is assumed to be some function of the present-day mass density (e.g. Coles 1993; Catelan et al. 1994; Weinberg 1995; Mann, Peacock \& Heavens 1998). The linear bias term is then the first interesting term in a Taylor expansion of the function about $\delta=0$. If the galaxy distribution is significantly biased with respect to the matter, there are major implications for cosmology, arising from the difficulty in detecting the matter distribution. On scales where masses can be reliably measured, such as in galaxy clusters, bias is required to reconcile the mass-to-light ratios with an Einstein-de Sitter Universe. Separately, linear studies of peculiar velocities, either directly (e.g. Sigad et al. 1998) or via redshift distortions (e.g. Hamilton 1992, Heavens \& Taylor 1995; Cole, Fisher \& Weinberg 1995; Hivon et al. 1995, Hamilton 1998, Hatton \& Cole 1998) return the density parameter of the Universe only via $\beta \equiv \Omega_{0}^{0.6} / b$, 
so ignorance of $b$ compromises determinations of $\Omega_{0}$. In addition to the Eulerian bias discussed in this paper, there are other possibilities, such as Lagrangian bias (e.g. Davis et al. 1985, Bardeen et al. 1986) and stochastic bias (Pen 1997; Tegmark \& Peebles 1998, Dekel \& Lahav 1998), biasing determined by halo properties at some redshift (Mo \& White 1996, Catelan et al. 1998, Catelan, Matarrese \& Porciani 1998), or biasing determined by coherent processes over a large scale (Babul \& White 1991; Bower et al. 1993). Despite a major industry in modelling (e.g. Kauffman et al. 1998), it is probably fair to say that we still have a great deal of uncertainty in precisely where galaxies should form, so it is not clear which, if any, of the above descriptions corresponds closely to reality. In this paper, we assume an Eulerian description for the bias, but we do not restrict it to linear form.

The current generation of galaxy redshift surveys, such as the Anglo-Australian Telescope 2dF survey (Colless 1996), and the Sloan Digital Sky Survey (Gunn \& Weinberg 1995) have sufficient space density and volume to look beyond linear theory, and this opens up possibilities of lifting the degeneracy between $\Omega_{0}$ and $b$, by measuring $b$ empirically through higher-order statistics (Matarrese, Verde \& Heavens 1997; Verde et al. 1998). Higher-order studies of the power spectrum have been made in real space by Makino, Sasaki \& Suto (1992), Jain \& Bertschinger (1994) and Baugh \& Efstathiou (1994), and also in an elegant treatment in real and redshift space in the Zel'dovich approximation by Taylor \& Hamilton (1996). Our treatment differs from previous perturbative calculations by its inclusion of bias and redshift distortions, and from the Zel'dovich approximation by inclusion of nonlinear, local bias and by the different treatment of nonlinear evolution. The layout of the paper is as follows: in Section 2 we set out the calculations formally, treating carefully the transition from real to redshift space; in Sections 3 and 4 we separate the major effects which come into operation beyond linear theory, in real and redshift space, and in Section 5 we present our conclusions.

\section{METHOD}

\subsection{Real to redshift space}

Let $\mathbf{r}$ and $\mathbf{s}$ be the real and redshift-space coordinates, with the observer at the origin. The latter is defined to be the recession velocity (including the peculiar velocities $\mathbf{v}$ of galaxy and observer) from the observer, divided by the Hubble constant $H_{0}$. $H_{0}=1$ is equivalent to using recession velocity as the distance coordinate, and we assume this from now on. Further we define $\rho_{r}(\mathbf{r})$ and $\rho_{s}(\mathbf{s})$ to be the density fields in real and redshift space. The mean density may be spatially-dependent, because of selection effects; we define the expected densities as $\phi_{r}(\mathbf{r})$ and $\phi_{s}(\mathbf{s})$. The overdensity in redshift space is defined by $1+\delta_{s}(\mathbf{s}) \equiv \rho_{s}(\mathbf{s}) / \phi_{s}(\mathbf{s})$, and similarly for the real-space overdensity $\delta_{r}$. In all, we shall work with four random fields:

- $\delta_{r}$ : mass overdensity in real space

- $\delta_{s}$ : mass overdensity in redshift space

- $\delta_{r}^{g}$ : galaxy overdensity in real space

- $\delta_{s}^{g}$ : galaxy overdensity in real space

and each will have its corresponding power spectrum $P_{s}^{g}$ etc. For simplicity and consistency with previous notation, we drop the $r$ subscript on the real space mass power spectrum.

The coordinate transform from real to redshift space is (Kaiser 1987)

$\mathbf{s}(\mathbf{r})=\mathbf{r}\left[1+\frac{U(\mathbf{r})-U(\mathbf{0})}{r}\right]$

where $U(\mathbf{r}) \equiv \mathbf{v} \cdot \mathbf{r} /\left(H_{0} r\right)$. Number conservation implies $\rho_{r}(\mathbf{r}) d^{3} \mathbf{r}=\rho_{s}(\mathbf{s}) d^{3} \mathbf{s}$, which gives

$\left[1+\delta_{s}(\mathbf{s})\right] \phi_{s}(\mathbf{s})=\frac{\left[1+\delta_{r}(\mathbf{r})\right] \phi_{r}(\mathbf{r})}{J}$

where the Jacobian is

$J=\left[1+\frac{\Delta U}{r}\right]^{2}\left(1+\frac{\partial U(\mathbf{r})}{\partial r}\right)$

and $\Delta U(\mathbf{r}) \equiv U(\mathbf{r})-U(\mathbf{0})$. We make now the large-distant-volume approximation, where we assume that any modes we analyse have wavenumbers $k$ which satisfy $k r \gg 1$ throughout. Terms $\Delta U / r$, which are $\sim \delta /(k r)$ if $\Omega \simeq 1$, are ignored entirely in comparison with $\delta$. If we assume that $\phi$ drops as some power of $r$, then a Taylor expansion of $\phi(s)$ yields $\phi(r)$ plus negligible correction terms. Beyond linear theory, the lowest-order contributions to the power spectrum arise from both second- and third-order terms in $\delta$, so we expand to third order:

$\delta_{s}(\mathbf{s})=\delta_{r}(\mathbf{r})-U^{\prime}(\mathbf{r})+U^{\prime 2}(\mathbf{r})-\delta_{r}(\mathbf{r}) U^{\prime}(\mathbf{r})+\delta_{r}(\mathbf{r}) U^{\prime 2}(\mathbf{r})-U^{\prime 3}(\mathbf{r}) \equiv F(\mathbf{r})$,

and ${ }^{\prime} \equiv \partial / \partial r$. To linear order, differences in the argument do not matter, but to third order, they do. To the required order, 
$F(\mathbf{r})=F(\mathbf{s})-\Delta U(\mathbf{s}) F^{\prime}(\mathbf{s})\left[1-U^{\prime}(\mathbf{s})\right]+\frac{1}{2} \Delta U^{2}(\mathbf{s}) F^{\prime \prime}(\mathbf{s})$,

which gives us the final expression for the redshift-space overdensity in terms of the real-space overdensity, all evaluated at s:

$\delta_{s}=\delta_{r}-U^{\prime}+U^{\prime 2}-\delta_{r} U^{\prime}+\delta_{r} U^{\prime 2}-U^{\prime 3}-\Delta U \delta_{r}^{\prime}+\Delta U U^{\prime \prime}-3 \Delta U U^{\prime} U^{\prime \prime}+2 \Delta U U^{\prime} \delta_{r}^{\prime}+\Delta U U^{\prime \prime} \delta_{r}+\frac{1}{2} \Delta U^{2} \delta_{r}^{\prime \prime}-\frac{1}{2} \Delta U^{2} U^{\prime \prime \prime}$.

\subsection{Bias and evolution}

We assume that the galaxy overdensity field $\delta^{g} \equiv \delta n / n$, where $n$ is the number density of galaxies, is related to the mass overdensity field $\delta$ via a local function. This plausible 'local Eulerian' bias model is chosen for simplicity and tractability, and has some support from simulation (e.g. Cen \& Ostriker 1992, Kauffmann, Nusser \& Steinmetz 1997). Of course other schemes are possible, such as Lagrangian bias (e.g. Catelan et al. 1998; see also Cole \& Kaiser 1989, Mo \& White 1996), or nonlocal bias (e.g. Bahcall \& West 1992, Bower et al. 1993, Matsubara 1995). We expand the local function as a Taylor series around $\delta_{r}=0$ (Fry \& Gaztanaga 1993):

$\delta^{g}(\mathbf{r})=\sum_{j=0}^{\infty} \frac{b_{j}}{j !} \delta_{r}^{j}$.

An unbiased galaxy field would have $b_{1}=1$ and all other bias parameters zero. Already there is a subtlety. We will truncate the expansion, which will only be a good approximation if the value of $\delta$ is typically much less than unity. Therefore our biasing assumption is that there is some smoothing scale for which (7) is a good approximation, and the fields above should be interpreted as the smoothed galaxy and mass density fields. For the perturbative expansion to be valid, we choose a smoothing scale large enough that the smoothed density field has small fluctuations (this will be quantified later). If we wish to smooth on a smaller scale, then a numerical approach is probably necessary (Mann, Peacock \& Heavens 1998). Note also that the galaxy distribution is of course a point process. We assume that the positions are determined by a Poisson sampling of a density field whose overdensity is $\delta_{g}$. We do not consider stochastic biasing (Pen 1997), and ignore shot noise in the power spectrum.

The next non-zero terms beyond linear theory will be of order 4 in $\delta_{r}$, so we need to keep terms up to $j=3$. Throughout we ignore the $b_{0}$ term, required to ensure that $\left\langle\delta_{r}^{g}\right\rangle=0$, since we will be interested in the spectral properties of the galaxy field, and drop all terms which contribute only to $\mathbf{k}=\mathbf{0}$.

Inserting (7) into (四), we get, to third order in $\delta_{r}$ :

$$
\begin{aligned}
\delta_{s}^{g}= & {\left[b_{1} \delta_{r}-U^{\prime}\right]+\left[\frac{b_{2}}{2} \delta_{r}^{2}+U^{\prime 2}-b_{1} \delta_{r} U^{\prime}-b_{1} \delta_{r}^{\prime} \Delta U+\Delta U U^{\prime \prime}\right]+\left[\frac{b_{3}}{6} \delta_{r}^{3}-\frac{b_{2}}{2} \delta_{r}^{2} U^{\prime}+b_{1} \delta_{r} U^{\prime 2}-U^{\prime 3}-b_{2} \Delta U \delta_{r} \delta_{r}^{\prime}\right.} \\
& \left.-3 \Delta U U^{\prime} U^{\prime \prime}+2 b_{1} \delta_{r}^{\prime} \Delta U U^{\prime}+b_{1} \delta_{r} \Delta U U^{\prime \prime}+\frac{1}{2} b_{1} \delta_{r}^{\prime \prime}(\Delta U)^{2}-\frac{1}{2}(\Delta U)^{2} U^{\prime \prime \prime}\right] .
\end{aligned}
$$

The Fourier transform of this may be taken in redshift space, making use of the transforms of simple products:

$$
\begin{aligned}
(X Y)_{\mathbf{k}} & =\frac{1}{(2 \pi)^{3}} \int d^{3} \mathbf{k}_{1} d^{3} \mathbf{k}_{2} \delta^{D}\left(\mathbf{k}-\mathbf{k}_{1}-\mathbf{k}_{2}\right) X_{1} Y_{2} \\
(X Y Z)_{\mathbf{k}} & =\frac{1}{(2 \pi)^{6}} \int d^{3} \mathbf{k}_{1} d^{3} \mathbf{k}_{2} d^{3} \mathbf{k}_{3} \delta^{D}\left(\mathbf{k}-\mathbf{k}_{1}-\mathbf{k}_{2}-\mathbf{k}_{3}\right) X_{1} Y_{2} Z_{3}
\end{aligned}
$$

where $X_{1}$ is the $\mathbf{k}_{1}$ component of $X_{\mathbf{k}}$ etc, and $\delta^{D}$ is the Dirac delta function. In the distant-observer approximation, $\partial / \partial r \rightarrow$ $i k \mu$, where $\mu \equiv \mathbf{k} \cdot \hat{\mathbf{r}} / k$ and $\hat{\mathbf{r}}$ is a unit vector from the observer to the galaxy (assumed constant across the sample) and the transform of $U$ is $U_{\mathbf{k}}=i \mu f \eta_{\mathbf{k}} / k$, where $-\eta_{\mathbf{k}}$ is the transform of the velocity divergence. $f \equiv d \ln D / d \ln a \simeq \Omega_{0}^{0.6}$, where $D(a)$ is the growing-mode amplitude and $a$ is the scale factor of the Universe. Second-order terms were computed by Peebles (1980); the third-order expansion of the fluid equations (see Fry 1984) is detailed in Catelan \& Moscardini (1994a) and Catelan \& Moscardini (1994b), giving $\delta_{\mathrm{k}}$ :

$$
\begin{aligned}
\delta_{\mathbf{k}} & =\epsilon_{\mathbf{k}}+\frac{1}{(2 \pi)^{3}} \int d^{3} \mathbf{k}_{1} d^{3} \mathbf{k}_{2} \delta^{D}\left(\mathbf{k}-\mathbf{k}_{1}-\mathbf{k}_{2}\right) J_{S}^{(2)}\left(\mathbf{k}_{1}, \mathbf{k}_{2}\right) \epsilon_{1} \epsilon_{2} \\
& +\frac{1}{(2 \pi)^{6}} \int d^{3} \mathbf{k}_{1} d^{3} \mathbf{k}_{2} d^{3} \mathbf{k}_{3} \delta^{D}\left(\mathbf{k}-\mathbf{k}_{1}-\mathbf{k}_{2}-\mathbf{k}_{3}\right) J_{S}^{(3)}\left(\mathbf{k}_{1}, \mathbf{k}_{2}, \mathbf{k}_{3}\right) \epsilon_{1} \epsilon_{2} \epsilon_{3}
\end{aligned}
$$

$\epsilon_{\mathbf{k}}$ is the linear, real-space Fourier coefficient of the density field $\delta(\mathbf{r})$. The corresponding expressions for $\eta_{\mathbf{k}}$ involve replacing $J$ by $K$. The functions $J$ and $K$ are quoted for $\Omega_{0}=1$, as computed by Goroff et al. (1986), Catelan \& Moscardini (1994a) 
and Catelan \& Moscardini (1994b):

$$
\begin{aligned}
J_{S}^{(2)}\left(\mathbf{k}_{1}, \mathbf{k}_{2}\right)= & \frac{5}{7}+\frac{\mathbf{k}_{1} \cdot \mathbf{k}_{2}}{2 k_{1} k_{2}}\left(\frac{k_{1}}{k_{2}}+\frac{k_{2}}{k_{1}}\right)+\frac{2}{7}\left(\frac{\mathbf{k}_{1} \cdot \mathbf{k}_{2}}{k_{1} k_{2}}\right)^{2}, \\
K_{S}^{(2)}\left(\mathbf{k}_{1}, \mathbf{k}_{2}\right)= & \frac{3}{7}+\frac{\mathbf{k}_{1} \cdot \mathbf{k}_{2}}{2 k_{1} k_{2}}\left(\frac{k_{1}}{k_{2}}+\frac{k_{2}}{k_{1}}\right)+\frac{4}{7}\left(\frac{\mathbf{k}_{1} \cdot \mathbf{k}_{2}}{k_{1} k_{2}}\right)^{2}, \\
J^{(3)}\left(\mathbf{k}_{1}, \mathbf{k}_{2}, \mathbf{k}_{3}\right)= & J^{(2)}\left(\mathbf{k}_{2}, \mathbf{k}_{3}\right)\left[\frac{1}{3}+\frac{1}{3} \frac{\mathbf{k}_{1} \cdot\left(\mathbf{k}_{2}+\mathbf{k}_{3}\right)}{\left(\mathbf{k}_{2}+\mathbf{k}_{3}\right)^{2}}+\frac{4}{9} \frac{\mathbf{k} \cdot \mathbf{k}}{k_{1}^{2}} \frac{\mathbf{k} \cdot\left(\mathbf{k}_{2}+\mathbf{k}_{3}\right)}{\left(\mathbf{k}_{2}+\mathbf{k}_{3}\right)^{2}}\right] \\
& -\frac{2}{9} \frac{\mathbf{k} \cdot \mathbf{k}_{1}}{k_{1}^{2}} \frac{\mathbf{k} \cdot\left(\mathbf{k}_{2}+\mathbf{k}_{3}\right)}{\left(\mathbf{k}_{2}+\mathbf{k}_{3}\right)^{2}} \frac{\left(\mathbf{k}_{2}+\mathbf{k}_{3}\right)}{k_{3}^{2}}+\frac{1}{9} \frac{\mathbf{k} \cdot \mathbf{k}_{2}}{k_{2}^{2}} \frac{\mathbf{k} \cdot \mathbf{k}_{3}}{k_{3}^{2}} \\
K^{(3)}\left(\mathbf{k}_{1}, \mathbf{k}_{2}, \mathbf{k}_{3}\right)= & 3 J^{(3)}\left(\mathbf{k}_{1}, \mathbf{k}_{2}, \mathbf{k}_{3}\right)-\frac{\mathbf{k} \cdot \mathbf{k}_{1}}{k_{1}^{2}} J^{(2)}\left(\mathbf{k}_{2}, \mathbf{k}_{3}\right)-\frac{\mathbf{k} \cdot\left(\mathbf{k}_{1}+\mathbf{k}_{2}\right)}{\left(\mathbf{k}_{1}+\mathbf{k}_{2}\right)^{2}} K^{(2)}\left(\mathbf{k}_{1}, \mathbf{k}_{2}\right),
\end{aligned}
$$

with $\mathbf{k}=\mathbf{k}_{1}+\mathbf{k}_{2}+\mathbf{k}_{3}$ in the last two expressions. The subscript $S$ indicates that the expression has been made symmetric w.r.t. any permutation of the arguments. If not, then the symmetrized kernel must be obtained by averaging the quoted expression over all permutations. These kernels are correct for $\Omega_{0}=1$ but are only weakly dependent on $\Omega_{0}$ (e.g. Bouchet et al. 1992, Catelan et al. 1995; see also Bernardeau 1994b, Eisenstein 1997, Kamionkowski \& Buchalter 1998). After some manipulations, the transform of the third-order, biased, redshift-space density field is

$$
\begin{aligned}
\delta_{s \mathbf{k}}^{g}= & F_{S}^{(1)}(\mathbf{k}) \epsilon_{\mathbf{k}}+\frac{1}{(2 \pi)^{3}} \int d^{3} \mathbf{k}_{1} d^{3} \mathbf{k}_{2} \delta^{D}\left(\mathbf{k}-\mathbf{k}_{1}-\mathbf{k}_{2}\right) F_{S}^{(2)}\left(\mathbf{k}_{1}, \mathbf{k}_{2}\right) \epsilon_{1} \epsilon_{2} \\
& +\frac{1}{(2 \pi)^{6}} \int d^{3} \mathbf{k}_{1} d^{3} \mathbf{k}_{2} d^{3} \mathbf{k}_{3} \delta^{D}\left(\mathbf{k}-\mathbf{k}_{1}-\mathbf{k}_{2}-\mathbf{k}_{3}\right) F_{S}^{(3)}\left(\mathbf{k}_{1}, \mathbf{k}_{2}, \mathbf{k}_{3}\right) \epsilon_{1} \epsilon_{2} \epsilon_{3}
\end{aligned}
$$

with the following kernels:

$$
\begin{aligned}
F_{S}^{(1)}(\mathbf{k})= & b_{1}+f \mu^{2} \\
F_{S}^{(2)}\left(\mathbf{k}_{1}, \mathbf{k}_{2}\right)= & b_{1} J_{S}^{(2)}\left(\mathbf{k}_{1}, \mathbf{k}_{2}\right)+f \mu^{2} K_{S}^{(2)}\left(\mathbf{k}_{1}, \mathbf{k}_{2}\right)+\frac{1}{2} b_{2}+\frac{b_{1} f}{2}\left[\mu_{1}^{2}+\mu_{2}^{2}+\mu_{1} \mu_{2}\left(\frac{k_{1}}{k_{2}}+\frac{k_{2}}{k_{1}}\right)\right] \\
& +f^{2}\left[\mu_{1}^{2} \mu_{2}^{2}+\frac{\mu_{1} \mu_{2}}{2}\left(\mu_{1}^{2} \frac{k_{1}}{k_{2}}+\mu_{2}^{2} \frac{k_{2}}{k_{1}}\right)\right] \\
F^{(3)}\left(\mathbf{k}_{1}, \mathbf{k}_{2}, \mathbf{k}_{3}\right)= & b_{1} J^{(3)}\left(\mathbf{k}_{1}, \mathbf{k}_{2}, \mathbf{k}_{3}\right)+f \mu^{2} K^{(3)}\left(\mathbf{k}_{1}, \mathbf{k}_{2}, \mathbf{k}_{3}\right)+\frac{b_{2}}{2} f \mu_{3}^{2}+\frac{b_{3}}{6}+\frac{b_{2}}{2} f \mu_{1} \mu_{2} \frac{k_{2}}{k_{1}}+\frac{b_{2}}{2} f \mu_{1} \mu_{3} \frac{k_{3}}{k_{1}}+b_{1} f^{2} \mu_{2}^{2} \mu_{3}^{2} \\
+ & 2 b_{1} f^{2} \mu_{1} \mu_{2} \mu_{3}^{2} \frac{k_{1}}{k_{2}}+b_{1} f^{2} \mu_{2} \mu_{3}^{3} \frac{k_{3}}{k_{2}}+\frac{b_{1}}{2} f^{2} \mu_{1}^{2} \mu_{2} \mu_{3} \frac{k_{1}^{2}}{k_{2} k_{3}}+f^{3} \mu_{1}^{2} \mu_{2}^{2} \mu_{3}^{2}+3 f^{3} \mu_{1} \mu_{2}^{2} \mu_{3}^{3} \frac{k_{3}}{k_{1}}+\frac{1}{2} f^{3} \mu_{1} \mu_{2} \mu_{3}^{4} \frac{k_{3}^{2}}{k_{1} k_{2}} \\
+ & J^{(2)}\left(\mathbf{k}_{2}, \mathbf{k}_{3}\right)\left(b_{2}+b_{1} f \mu_{1}^{2}+b_{1} f \mu_{1} \mu_{2+3} \frac{k_{2+3}}{k_{1}}\right) \\
+ & K^{(2)}\left(\mathbf{k}_{2}, \mathbf{k}_{3}\right)\left(b_{1} f \mu_{2+3}^{2}+b_{1} f \mu_{1} \mu_{2+3} \frac{k_{1}}{k_{2+3}}+2 f^{2} \mu_{1}^{2} \mu_{2+3}^{2}+f^{2} \mu_{1} \mu_{2+3}^{3} \frac{k_{2+3}}{k_{1}}+f^{2} \mu_{1}^{3} \mu_{2+3} \frac{k_{1}}{k_{2+3}}\right) .
\end{aligned}
$$

A subscript $S$ on the $F$ terms also indicates that the term has been symmetrized w.r.t. its arguments. Note that the last term has not been symmetrized and $\mu_{2+3} \equiv\left(\mathbf{k}_{2}+\mathbf{k}_{3}\right) \cdot \hat{\mathbf{r}} /\left|\mathbf{k}_{2}+\mathbf{k}_{3}\right|$.

From these equations we can obtain the power spectrum to third-order, which includes non-zero correction terms of two types, as in the real-space unbiased case (Makino, Sasaki \& Suto 1992; Jain \& Bertschinger 1994). The redshift-space power spectrum $P_{s}^{g}(\mathbf{k})$ is defined by

$\left\langle\delta_{s \mathbf{k}_{1}}^{g} \delta_{s \mathbf{k}_{2}}^{g}\right\rangle=(2 \pi)^{3} P_{s}^{g}\left(\mathbf{k}_{1}\right) \delta^{D}\left(\mathbf{k}_{1}+\mathbf{k}_{2}\right)$

and the real-space mass linear power spectrum $P_{11}(k)$ is defined similarly by $\left\langle\epsilon_{\mathbf{k}_{1}} \epsilon_{\mathbf{k}_{2}}\right\rangle=(2 \pi)^{3} P_{11}\left(k_{1}\right) \delta^{D}\left(\mathbf{k}_{1}+\mathbf{k}_{2}\right)$. The Gaussian nature of the initial fluctuations implies, by Wick's theorem, that $\left\langle\epsilon_{1} \epsilon_{2} \epsilon_{3}\right\rangle=0$ and $\left\langle\epsilon_{1} \epsilon_{2} \epsilon_{3} \epsilon_{4}\right\rangle=\left\langle\epsilon_{1} \epsilon_{2}\right\rangle\left\langle\epsilon_{3} \epsilon_{4}\right\rangle$ plus cyclic permutations. Hence we find two (one-loop) terms which arise at the next level beyond linear theory for the spectrum (tree level):

$$
\begin{aligned}
P_{s}^{g}(\mathbf{k}) & \equiv P_{s 11}^{g}+P_{s 22}^{g}+P_{s 13}^{g} \\
& =\left(1+\beta \mu^{2}\right)^{2} b_{1}^{2} P_{11}(k)+2 \int \frac{d^{3} \mathbf{q}}{(2 \pi)^{3}} P_{11}(q) P_{11}(|\mathbf{k}-\mathbf{q}|)\left[F_{S}^{(2)}(\mathbf{q}, \mathbf{k}-\mathbf{q})\right]^{2} \\
& +6\left(1+\beta \mu^{2}\right) b_{1} P_{11}(k) \int \frac{d^{3} \mathbf{q}}{(2 \pi)^{3}} P_{11}(q) F_{S}^{(3)}(\mathbf{q},-\mathbf{q}, \mathbf{k})
\end{aligned}
$$


where $\beta \equiv f / b_{1}$. In the unbiased case in real space $(\beta=0)$, these formulae reduce to those in Jain \& Bertschinger (1994) (note there is a factor of $k^{2}$ missing in their equation 19). For this case, the limits of integration for the $P_{22}^{g}$ and $P_{13}^{g}$ terms are not too important, provided the power spectrum decays sufficiently rapidly at high $k$ (faster than $k^{-1}$ ), and this is fine for spectra of practical interest. For the bias terms, however, one has to be careful, because if one places a cutoff in the integrals, one finds that the resulting power spectrum is sensitive to this cutoff. This should not be surprising: the cutoff effectively smoothes the linear power spectrum (with a top-hat filter in $k$-space), and the power spectrum depends on what scale the filtering takes place. Furthermore, it makes physical sense: if a local bias prescription applies at all, it will be necessary to specify on what scale it acts. These considerations lead us to the following Eulerian bias proposal:

- Evolve $\delta$ to 3rd-order

- Smooth with some filter function corresponding to a smoothing scale $R_{f}$

- Apply the Taylor expansion to third-order to get $\delta_{g}$.

The resulting field is evidently itself smoothed. This procedure is effected by replacing all occurrences of the linear $\epsilon_{k}$ by $\epsilon_{k} W_{k}$, where $W_{k}$ is the transform of the filter function [e.g. $W_{k}=\exp \left(-k^{2} R_{f}^{2} / 2\right)$ for a Gaussian filter]. We shall not explicitly retain these factors of $W_{k}$; the smoothing is implicit in $\epsilon_{k}$. Note that the operations of smoothing and biasing do not commute; unless the matter power spectrum has a natural small-scale cutoff, the order assumed here is the only sensible choice for a perturbative calculation. This is a critical assumption, of course, but we reiterate that it has some support from simulation (e.g. Cen \& Ostriker 1992). For further discussions of the effects of smoothing, see Coles, Melott \& Shandarin (1993), Melott \& Shandarin (1993), Bernardeau (1994a).

\subsection{Bispectrum}

The galaxy redshift-space bispectrum $B_{s}^{g}\left(\mathbf{k}_{1}, \mathbf{k}_{2}, \mathbf{k}_{3}\right)$ is the 3-point function in Fourier space, defined by $\left\langle\delta_{s \mathbf{k}_{1}} \delta_{s \mathbf{k}_{2}} \delta_{s \mathbf{k}_{3}}\right\rangle=(2 \pi)^{3} B_{s}^{g}\left(\mathbf{k}_{1}, \mathbf{k}_{2}, \mathbf{k}_{3}\right) \delta^{D}\left(\mathbf{k}_{1}+\mathbf{k}_{2}+\mathbf{k}_{3}\right)$.

In real-space, this has been studied by Matarrese, Verde \& Heavens (1997), Scoccimarro (1997) and Scoccimarro et al. (1998). We can use the preceding formalism to obtain the biased, redshift-space bispectrum, in the distant observer approximation:

$$
\begin{aligned}
B_{s}^{g}\left(\mathbf{k}_{1}, \mathbf{k}_{2}, \mathbf{k}_{3}\right) & =2 P_{11}\left(k_{1}\right) P_{11}\left(k_{2}\right) F_{S}^{(1)}\left(\mathbf{k}_{1}\right) F_{S}^{(1)}\left(\mathbf{k}_{2}\right) F_{S}^{(2)}\left(\mathbf{k}_{1}, \mathbf{k}_{2}\right)+\operatorname{cyclic} \text { terms }(1,2) \rightarrow(2,3) \text { and }(3,1) \\
& =2 P_{11}\left(k_{1}\right) P_{11}\left(k_{2}\right) b_{1}^{3}\left(1+\beta \mu_{1}^{2}\right)\left(1+\beta \mu_{2}^{2}\right)\left[J^{(2)}\left(\mathbf{k}_{1}, \mathbf{k}_{2}\right)+\beta \mu_{2} K^{(2)}\left(\mathbf{k}_{1}, \mathbf{k}_{2}\right)+\frac{b_{2}}{2 b_{1}}+b_{1} \beta^{2} \mu_{1}^{2} \mu_{2}^{2}+\frac{b_{1} \beta}{2}\left(\mu_{1}^{2}+\mu_{2}^{2}\right)\right. \\
& \left.+\frac{b_{1} \beta}{2} \mu_{1} \mu_{2}\left(\frac{k_{1}}{k_{2}}+\frac{k_{2}}{k_{1}}\right)+\frac{b_{1}^{2}}{2} \beta^{2} \mu_{1} \mu_{2}\left(\mu_{1}^{2} \frac{k_{1}}{k_{2}}+\mu_{2}^{2} \frac{k_{2}}{k_{1}}\right)\right]+ \text { cyclic terms. }
\end{aligned}
$$

In a separate paper (Verde et al. 1998) we use this to investigate how redshift surveys can be used practically to estimate $b_{1}$ and hence determine the density parameter (via $\beta$ ).

\section{NEW FEATURES: REAL SPACE}

Interestingly, there are some new features which arise in real-space from biasing alone. Consider the real-space corrections to the power spectrum, $P_{22}^{g} \equiv P_{s 22}^{g}(\beta=0)$ and $P_{13}^{g} \equiv P_{s 13}^{g}(\beta=0)$. At small $k$, the former is more-or-less constant with k, while the latter is proportional to $P_{11}(k)$. We look at each of these in turn.

\subsection{Constant power on very large scales}

On very large scales, where $\Delta^{2}(k) \ll 1$ (where $\Delta^{2}$ is the contribution to the variance in mass overdensity per unit $\ln (k)$ ), the terms arising from nonlinear evolution (and a linear bias $b_{1}$ ) are generally small (Jain \& Bertschinger 1994), so we concentrate on the contributions from nonlinear bias.

The $P_{22}^{g}$ term is

$P_{22}^{g}(k)=\frac{1}{4 \pi^{3}} \int d^{3} \mathbf{k}_{1} P\left(\mathbf{k}_{1}\right) P\left(\left|\mathbf{k}-\mathbf{k}_{1}\right|\right)\left[b_{1} J_{s}^{(2)}\left(\mathbf{k}_{1}, \mathbf{k}-\mathbf{k}_{1}\right)+\frac{b_{2}}{2}\right]^{2}$

which provides a constant contribution to the power when $|\mathbf{k}|$ is small. The presence of such a term was suggested by Coles (1993) and Strauss \& Willick (1995); here we provide a mechanism for calculating it perturbatively for arbitrary local bias. 


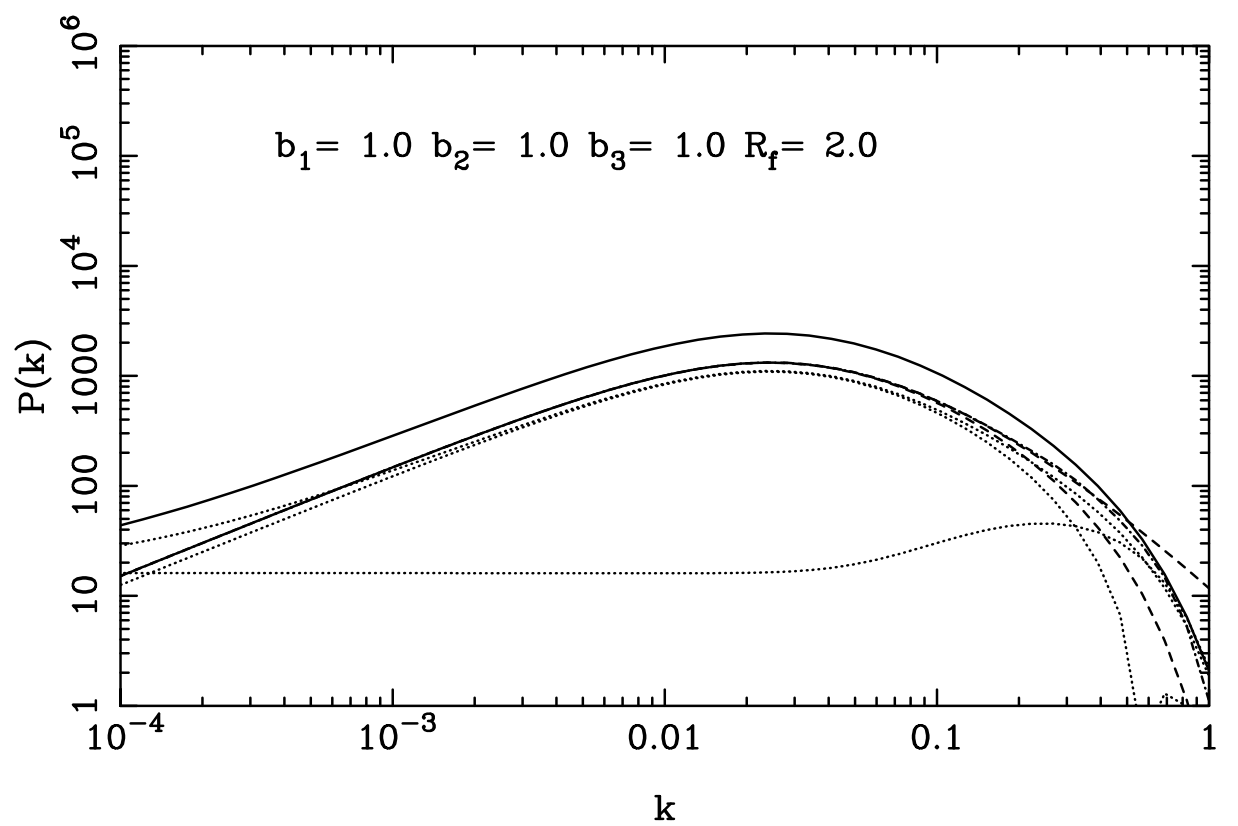

Figure 1. Real-space power spectrum. The (upper) solid line is the final power spectrum; the dashed lines are the linear power spectrum, unsmoothed and smoothed, and the dotted lines are (from the bottom at $k=10^{-3}$ ) the contributions to the power from $P_{22}, P_{13}$ and their sum. The dot-dashed line is the second-order mass power spectrum, which merges with the dashed linear power spectrum to form the lower solid line. For model details, see text.

For an underlying matter power spectrum which falls to zero as $k \rightarrow 0, P_{22}^{g}$ will eventually dominate over both the linear term and $P_{13}^{g}$, leading to a divergent effective bias $b_{\text {eff }}^{2}(k) \equiv P^{g}(k) / P(k)$ in this limit $(P$ is the matter power spectrum evolved to third order; on these large scales, linear theory is accurate, so $\left.P \simeq P_{11}\right)$. In turns out, for an underlying Zel'dovich spectrum, this term is unlikely to be of importance unless the other correction term $P_{13}^{g}$ below is small. The second-order power spectrum is shown in Fig. 1 for a CDM-like spectrum with shape parameter $\Gamma=0.25$, with and the amplitude is chosen such that $\Delta^{2}(k=0.1)=0.03$. The mass field is smoothed on a scale of $2 h^{-1} \mathrm{Mpc}$, so that the linear smoothed variance is $\sigma_{0}^{2}=0.20$, and the bias parameters are $b_{1}=b_{2}=b_{3}=1$. Note that the nonlinear bias masks the effect seen in the unbiased case by Jain \& Bertschinger (1994) of a transfer of power from large to small scales.

The constant power at large scales should not be confused with the requirement that $P(k) \rightarrow 0$ as $k \rightarrow 0$, which applies if the mean density is estimated from the survey. This will suppress the power for $k \lesssim 1 / L$, where $L$ is the characteristic depth of the survey, and is quite independent of the considerations here. Similarly, the neglect of constant terms in $\delta$ affects only $\mathbf{k}=\mathbf{0}$.

The term here has been conjectured before, and it is appropriate to make a few remarks about its nature. It is essentially a shot-noise term arising from the peaks and troughs in the underlying density field being nonlinearly-biased by the quadratic term $b_{2}$. The biasing gives a contribution to the density field consisting of spikes at the peaks and troughs. We consider here the case when the underlying matter power spectrum tends to zero on large scales, so we look only at this remaining 'spike' contribution (see Appendix A). It may seem odd that the troughs make a contribution; is should be remembered that they do not necessarily correspond to actual overdensities, as there is a linear contribution which generally lowers the density a positive quadratic term merely means the underdense regions are not quite as underdense as a linear bias would imply. However, by assumption, this linear contribution has zero power on very large scales, so the quadratic term dominates.

To illustrate the shot-noise nature of the spikes, consider a non-pathological power spectrum, filtered to remove power with $k>k_{\max }$. It is straightforward to show that the constant-power term is roughly

$P_{22}^{g} \simeq b_{2}^{2} \Delta^{4}\left(k_{\max }\right) \frac{1}{\bar{n}}$

where $\Delta\left(k_{\max }\right)$ measures the nonlinearity on the smoothing scale, and $\bar{n} \sim k_{\max }^{-3}$ is the number density of peaks and troughs on this scale (Peacock \& Heavens 1985, Bardeen et al. 1986). Shot noise of a point process gives $P_{\text {shot }}=1 / \bar{n}($ e.g. Peebles 1980$)$; the prefactor arises here because each spike has a height of roughly $b_{2} \sigma_{0}^{2}$ so the integral under each spike is $\sim b_{2} \Delta^{2}\left(k_{\text {max }}\right)$.

This shot noise argument would be dangerous if it was applied to peaks alone, as we know that these are strongly clustered (Kaiser 1984, Peacock \& Heavens 1985, Bardeen et al. 1986), and their clustering might well exceed the shot noise 


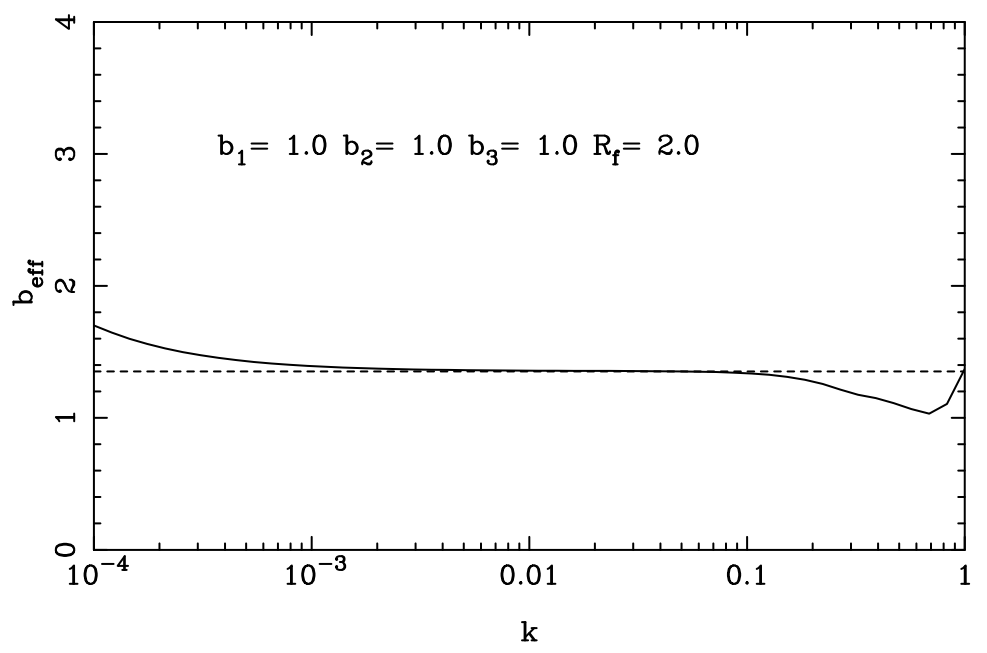

Figure 2. Effective bias in real space, from perturbation theory (solid). This is approximately constant over a fairly wide range of $k$, yet the bias is far from linear. The dotted line shows the approximate analytic formula (23). The filter erases power at $k \gtrsim 0.2$ and the spike at $k=1$ is due to the matter power spectrum crossing zero, by which time third-order perturbation theory has broken down.

contribution. In the appendix we prove that high peaks and low troughs, taken together, are very weakly clustered, so the power contribution is indeed essentially a shot noise term from unclustered small-scale spikes in the biased field.

\subsection{Effective bias on large scales is not $b_{1}$}

If the $P_{22}^{g}$ term can be neglected in comparison with $P_{13}^{g}$, then the effect of biasing and second-order evolution is to add a term which is proportional to the linear $P$. This holds over a wide range of scales, but not at the very largest scales, where the power spectrum is dominated by the constant contribution from $P_{22}^{g}$, leading to a divergent bias.

The fact that $P_{13}^{g}$ is proportional to $P_{11}$ gives a constant effective bias $\left[b_{\text {eff }}^{2} \equiv P^{g}(k) / P(k) \simeq P^{g}(k) / P_{11}(k)\right]$. In linear theory, the effective bias is simply $b_{1}$; in this section we compute the corrections to this value. In real space, only 3 terms of $F^{(3)}$ survive. The first, proportional to $J^{(3)}$ comes from nonlinear evolution of the mass field, and is very small on large scales, and we neglect it here. There is a straightforward contribution from $b_{3} / 6$, which gives

$b_{1} b_{3} P_{11}(k) \sigma_{0}^{2}$

where $\sigma_{0}^{2}=\left(2 \pi^{2}\right)^{-1} \int d k k^{2} P_{11}(k)$ is the linear variance in the smoothed field. The other nonzero term is

$\frac{2 b_{2}}{(2 \pi)^{3}} \int d^{3} \mathbf{k} P_{11}(\mathbf{q})\left[J_{S}^{(2)}(-\mathbf{q}, \mathbf{k})+J_{S}^{(2)}(\mathbf{q}, \mathbf{k})+J_{S}^{(2)}(\mathbf{q},-\mathbf{q})\right]$

The last term in brackets is zero because $J$ is zero, and the first terms are equal. Integration over angles gives a contribution again proportional to $\sigma_{0}^{2}$, and the final result is

$P_{13}^{g}(k)=b_{1} P_{11}(k)\left(\frac{68}{21} b_{2}+b_{3}\right) \sigma_{0}^{2}$.

The $P_{13}^{g}$ term is of more practical importance than $P_{22}^{g}$. We see that it is proportional to the linear power spectrum, so it corresponds to a scale-independent bias, which, when including the linear terms, gives an effective bias of

$b_{\text {eff }}^{2}(k)=b_{1}^{2}+b_{1}\left(\frac{68}{21} b_{2}+b_{3}\right) \sigma_{0}^{2}$.

The level of bias depends on the variance of the smoothed field, for which the truncated Taylor expansion is assumed to be a good description of the galaxy density field. It also depends on both the second- and the third-order bias coefficients. The suggestion that the bias should be constant on large scales has been made by a number of authors (Coles 1993; Scherrer \& Weinberg 1997; Mann, Peacock \& Heavens 1998), and we confirm that this is true to third order in perturbation theory, provided one is not looking at the very largest scales where $P_{22}^{g}$ dominates.

The effective bias is plotted in Fig. 2, along with the prediction of (23), which is seen to be a good approximation over a wide range of $k$, for these parameters. In Fig. 3 we demonstrate that the approximate formula works well for a biased N-body simulation, with the same linear power spectrum, normalised to $\sigma_{8}=0.26$, from the Hydra consortium (Couchman, Thomas \& Pearce 1995). The rather small amplitude of the power spectrum gives us a large dynamic range between the smoothing 


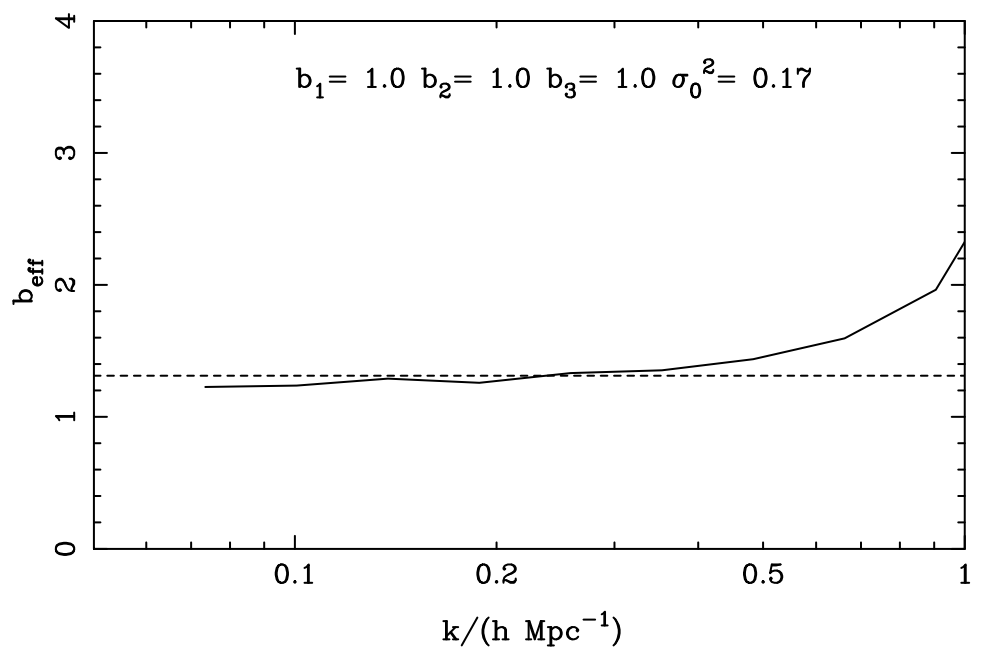

Figure 3. The effective bias factor $\sqrt{P_{\text {biased }} / P_{\text {mass }}}$ for a Hydra N-body simulation (solid), along with the approximate formula (23), for $b_{1}=b_{2}=b_{3}=1$ and $R_{f}=2 h^{-1} \mathrm{Mpc}$, which gives an r.m.s. fractional overdensity of 0.41 . For other details, see text. This figure also differs from Fig. 2 in that the biased field has been deconvolved with a Gaussian, which affects the results at high $k$.

scale and the size of the simulation. The smoothing is done with a Gaussian filter of radius $1 h^{-1} \mathrm{Mpc}$, giving a variance of $\sigma_{0}^{2}=0.17$, and we take $b_{1}=b_{2}=b_{3}=1$. Note that a bias function which includes non-zero $b_{i}$ terms beyond $i=3$, such as the exponential high-peak biasing $\exp (\alpha \delta)$ (Politzer \& Wise 1984, Jensen \& Szalay 1986, Bardeen et al. 1986) gives essentially the same result provided that the variance in the field is not too large. For the power spectra here, this requires $\alpha \sigma_{0} \lesssim 0.3$, but the exact figure depends on how skewed the field is. As the field becomes progressively more nonlinear, the bias increases slightly towards high $k$, and the approximate formula (23) begins to underestimate the bias. Note that the simulations do not probe a large enough scale to detect the constant contribution to the power.

Since $P_{13}^{g}$ is proportional to the linear power, it is formally possible at this level of perturbation theory to cancel the linear power altogether by appropriate choice of $b_{2}$ and $b_{3}$, leaving the constant $P_{22}^{g}$ term. Perhaps not surprisingly, it appears impossible to do this on N-body simulations without perturbation theory breaking down.

\section{REDSHIFT-SPACE}

In Fig. 4 we show the effects in redshift space of biasing and evolution, for different angles of wavevectors with the line-ofsight, showing similar qualitative behaviour to Kaiser's (1984) analysis. In Fig. 5 we show the redshift-space power spectrum (averaged over angle) for the Hydra simulation, for the same smoothings and parameters as in Fig. 3. We see here remarkably good agreement with perturbation theory of biased fields in redshift space. Note that linear theory, with linear bias, predicts that the redshift-space and real-space power spectra should be proportional to each other. Here we see that the higher-order terms give very different behaviour. Note also that perturbation theory gives excellent results all the way down to Mpc scales, where the smoothing is applied.

\subsection{Quadrupole-to-monopole ratio}

Hamilton (1992) proposed the quadrupole-to-monopole ratio of the power spectrum as a diagnostic for $\beta$. These are obtained by expansion of the power spectrum in a shell of fixed $|\mathbf{k}|$, in terms of Legendre polynomials (in $\mu$ ) of order 0 and 2 . In linear theory, the ratio of the coefficients $\mathcal{P}_{i}^{s}$ has the value

$\frac{\mathcal{P}_{2}^{s}}{\mathcal{P}_{0}^{s}}=\frac{\frac{4}{3} \beta+\frac{4}{7} \beta^{2}}{1+\frac{2}{3} \beta+\frac{1}{5} \beta^{2}}$.

Taylor \& Hamilton (1996) extended this idea by using the Zel'dovich approximation to investigate the behaviour of the quadrupole-to-monopole ratio into the mildly nonlinear regime. In this section we use perturbation theory results to investigate the influence that bias might have on conclusions drawn from the ratio. In practice, it is best to use the full 3D power spectrum to estimate parameters, but analysis of the quadrupole-to-monopole ratio can be a useful aid to see why we might be able to extract information, and why there might be problems. In Fig. 6 we see that, on large scales, linear theory accounts well for the ratio, with significant deviations appearing on small scales in qualitative agreement with Taylor \& Hamilton (1996). The 


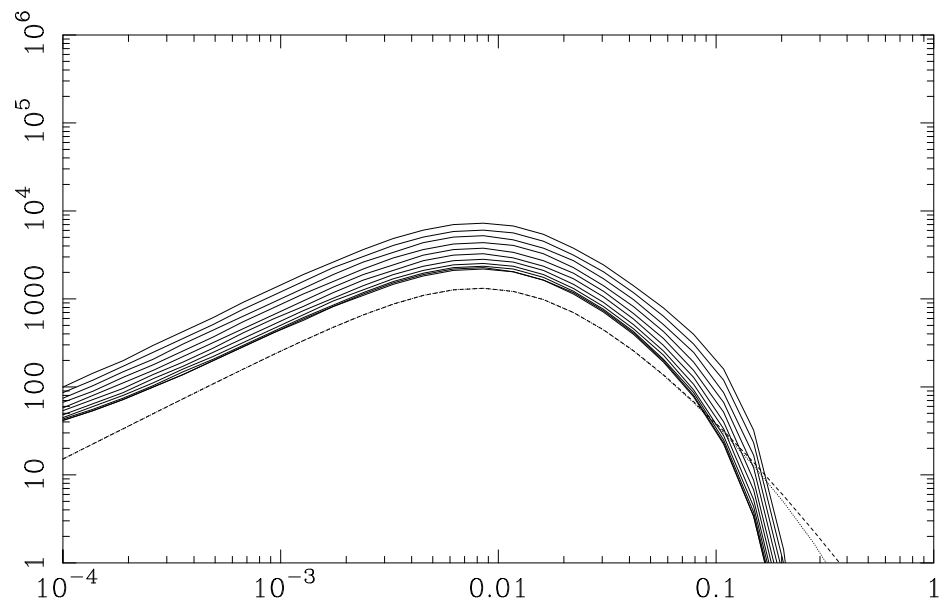

Figure 4. Redshift-space power spectrum for the model of Fig. 1 . The curves are for values of $\mu$ from 0 (bottom) to 1 in steps of 0.1 . The dashed and dotted lines are the unsmoothed linear and nonlinear power spectrum in real space.

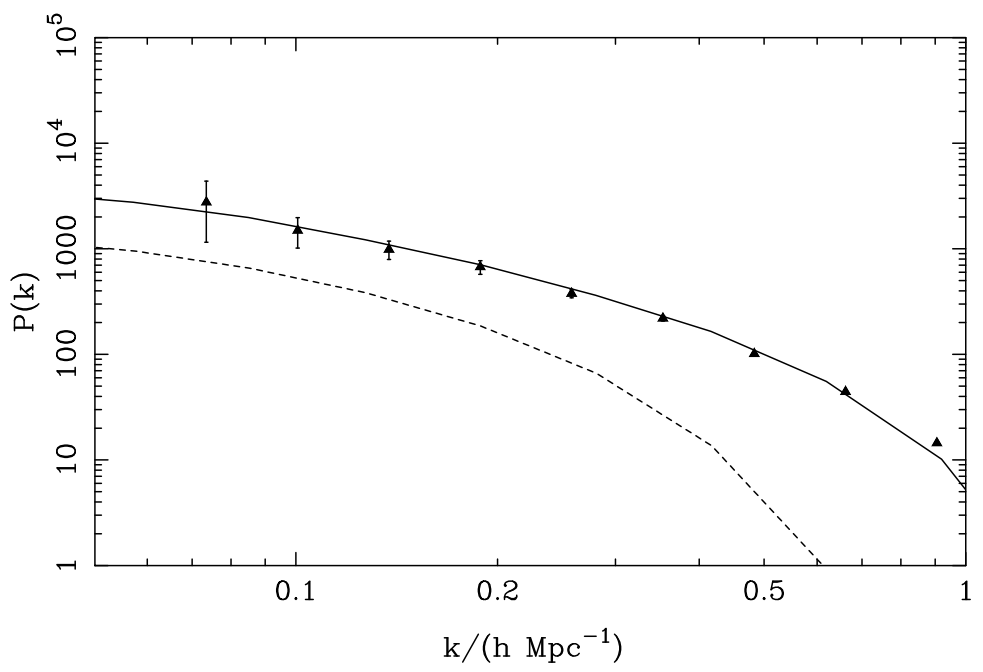

Figure 5. Hydra simulations in redshift space (points), along with the theoretical curve from perturbation theory $\left(b_{1}=b_{2}=b_{3}=1.0\right.$, and $\sigma_{0}=0.41$ ). The dotted line is the smoothed linear theory matter power spectrum in real space.

slight discrepancy with linear theory may arise from only computing the power spectrum with a rather crude separation of $\Delta \mu=0.1$. On small scales, the quadrupole-to-monopole ratio was studied by Taylor \& Hamilton (1996) and Hatton \& Cole (1998), where it is affected seriously by caustic formation and small-scale virialised structures. We confine our remarks to larger scales $(k \lesssim 0.1)$, where these effects are not important, but there are still some surprises. Fig. 7 shows the quadrupoleto-monopole ratio for a biased CDM-like field, with $\Gamma=0.25$, with $b_{1}=b_{2}=b_{3}=1.0$ and $\sigma_{0}^{2}=0.196$ and $R_{f}=2 h^{-1}$ Mpc. There is a large range of wavenumbers for which the ratio is very nearly constant, which might give one confidence that a linear bias is an adequate description. However, the retrieved value of $\beta=0.81$ agrees neither with linear theory $(\beta=1)$, nor with the appropriate $\beta$ from the effective bias on large scales $(\beta=0.74)$. It is the latter which we would like to estimate accurately, since it is the effective bias which tells us the amplitude of the underlying matter power spectrum. In this case, the recovered effective bias (1.24) is not close to the true effective bias (1.35). The discrepancy arises because the one-loop corrections do not have the same angular dependence as the Kaiser factor multiplying the linear power. This is not really a breakdown of linear theory as such; the underlying mass field is still well described by linear perturbation theory. Here it is the nonlinear bias which causes the problem, and the simple analysis of Kaiser (1987) cannot be applied. These findings mean that attempts to measure $\beta$ from linear redshift distortions (e.g. Hamilton 1992; Fisher, Scharf \& Lahav 1994; Heavens \& Taylor 1995; Ballinger, Heavens \& Taylor 1995; Cole, Fisher \& Weinberg 1995; Tadros et al. 1998) must make the further assumption that the one-loop corrections are small. 


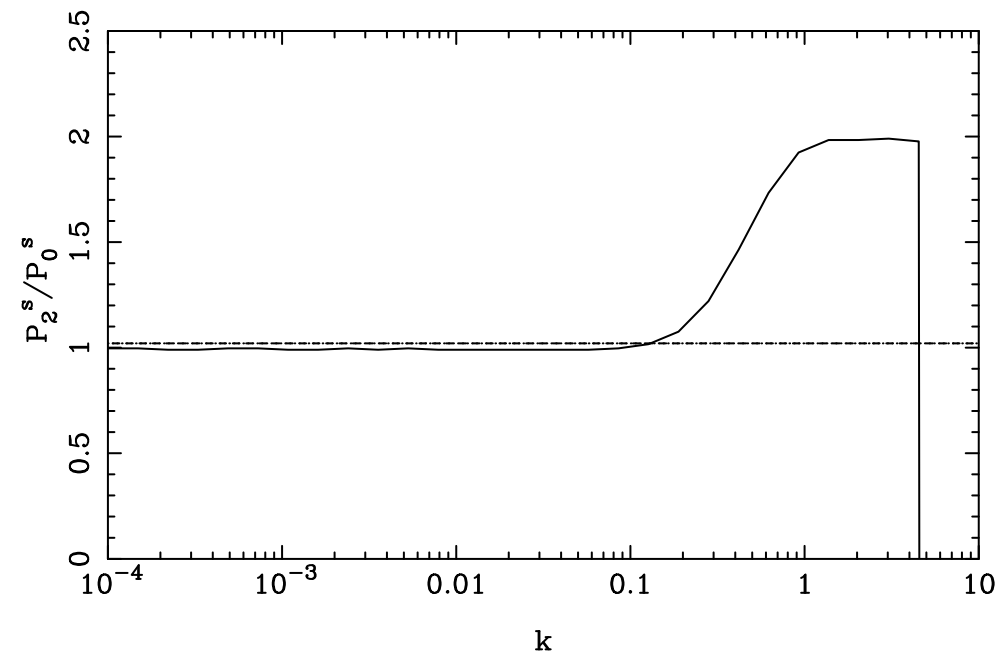

Figure 6. Quadrupole-to-monopole ratio for the mass, as a function of wavenumber (solid). Beyond $k=4.5$, the filtered power is zero to machine accuracy. The dashed line is the linear theory ratio (24).

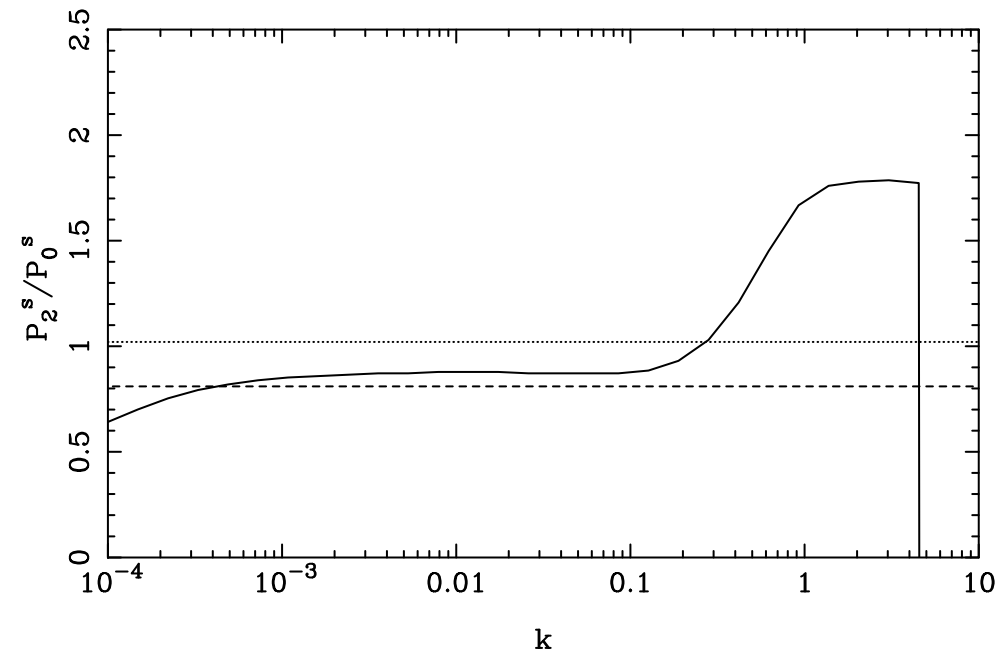

Figure 7. Quadrupole-to-monopole ratio of the biased field (solid), as a function of wavenumber, along with unbiased linear theory (dotted) and linear theory, but with $b$ replaced by $b_{\text {eff }}$ from perturbation theory (dashed). The behaviour at $k<10^{-3}$ arises from the constant-power term in the biased field.

\section{CONCLUSIONS}

In this paper, we have shown how the power spectrum is altered in real and redshift space by the effects of nonlinear local bias and evolution. The assumptions in the analysis are that the smoothed galaxy density field is some local function of the underlying smoothed present-day mass field (i.e. Eulerian local bias). The smoothing scale is assumed to be large enough that a Taylor expansion to third order: $\delta_{g} \simeq \sum_{j=0}^{3} b_{j} \delta^{j} / j$ ! suffices. The approach we have used is perturbative; the one-loop corrections give contributions from third-order (in $\delta$ ) which are as important as second order, so we have kept evolution and bias terms to this order $(j=3)$. We confirm the predictions of Scherrer \& Weinberg (1997) that the power spectrum has a constant component, plus a roughly scale-independent bias on large scales. We show that the constant power term is essentially a shot noise term arising from quadratic biasing of peaks and troughs. The most important results in real space are the expressions for the magnitudes of the two effects: equation (23) for the effective bias on large scales, and (18) for the constant contribution, which could in principle make difficult the task of unveiling the underlying power spectrum on extremely large scales. However, our estimates of the size of this effect suggest that it is not going to be a practical problem in the foreseeable future, since it is likely to dominate only on scales of the order of the horizon size. Should the galaxy power spectrum be measured on extremely large scales at some time in the future, there is a possibility that a deviation from the 
scale-invariant spectrum (in $|k|$ ) might be interpreted as evidence for an open Universe (Kamionkowski \& Spergel 1994). This paper provides an alternative interpretation in terms of nonlinear bias.

Potentially more serious is the effect of nonlinear bias on the amplitude of the power spectrum, in both real and redshift space. Here there is the possibility of confusion, in that the shape of the linear power spectrum is preserved over a wide range of $k$, so one can define a scale-independent effective bias from the ratio of the galaxy to matter power spectra. The difficulty is that this bias is not the linear bias ( $b_{1}$ in the Taylor expansion of the galaxy density field), nor is it the bias which would be recovered from studying anisotropy in the redshift power spectrum. These complications may compromise efforts to deduce cosmological parameters from redshift distortions or peculiar velocities, unless a further assumption is made that the one-loop corrections are small. Without a better understanding of galaxy formation, it is impossible to make this assumption with a great deal of confidence. What would be interesting to see is whether current models can identify a scale on which an Eulerian bias is a good description of the galaxy field; if such a scale can be identified, then the computations in this paper may be useful in providing analytic approximations for the effects on the power spectrum and redshift distortion.

\section{ACKNOWLEDGMENTS}

LV acknowledges support from the European Union for support under the TMR programme. AFH thanks the University of Padova, and SM the University of Edinburgh for hospitality. Calculations were performed on STARLINK facilities. The simulations were obtained from the data bank of cosmological N-body simulations provided by the Hydra consortium (http://coho.astro.uwo.ca/pub/data.html) and produced using the Hydra N-body code (Couchman et al. 1995). We thank Francesco Lucchin, Lauro Moscardini, Bhuvnesh Jain and Andy Taylor for useful discussions.

\section{REFERENCES}

Babul A., White S. D. M., 1991.MNRAS, 253, 31P.

Bahcall N. A., West M., 1992. ApJ, 392, 419.

Ballinger W. E., Heavens A. F., Taylor A. N., 1995. MNRAS, 276, 59P.

Bardeen J. M., Bond J. R., Kaiser N., Szalay A. S., 1986. ApJ, 304, 15.

Baugh C. M., Efstathiou G., 1994. MNRAS, 270, 183.

Bernardeau F., 1994a. A\& A, 291, 697.

Bernardeau F., 1994b. ApJ, 433, 1.

Bouchet F., Juszkiewicz R., Colombi S., Pellat R., 1992. ApJ, 394, L5.

Bower R. G., Coles P., Frenk C., White S., 1993. ApJ, 405, 403.

Catelan P., Moscardini L., 1994a. ApJ, 426, 14.

Catelan P., Moscardini L., 1994b. ApJ, 436, 5.

Catelan P., Coles P., Matarrese S., Moscardini L., 1994.MNRAS, 268, 966.

Catelan P., Lucchin F., Matarrese S., Moscardini L., 1995. MNRAS, 276, 39.

Catelan P., Lucchin F., Matarrese S., Porciani C., 1998. MNRAS, 297, 692.

Catelan P., Matarrese S., Porciani C., 1998. ApJ(Lett), in press (astroph/9804250).

Cen R. Y., Ostriker J. P., 1992. ApJ(Lett), 399, L13.

Cole S., Kaiser N., 1989. MNRAS, 237, 1127.

Cole S., Fisher K. B., Weinberg D. H., 1995. MNRAS, 275, 515.

Coles P., Melott A., Shandarin S., 1993.MNRAS, 260, 765.

Coles P., 1993. MNRAS, 262, 1065.

Colless M., 1996.In: Wide Field Spectroscopy, 227, eds Kontizas M., Kontizas E., Kluwer.

Couchman H. M. P., Thomas P. A., Pearce F. R., 1995. ApJ, 452, 797.

Davis M., Efstathiou G., Frenk C. S., White S. D. M., 1985.ApJ, 292, 371.

Dekel A., Lahav O., 1998. astro-ph, 9806193.

Eisenstein D., 1997. astro-ph, 9709054.

Fisher K. B., Scharf C., Lahav O., 1994.MNRAS, 266, 219.

Fry J. N., Gaztanaga E., 1993. ApJ, 413, 447.

Fry J. N., 1984. ApJ, 279, 499.

Goroff M. H., Grindstein B., Rey S. J., Wise B., 1986. ApJ, 311, 6.

Gunn J. E., Weinberg D. H., 1995. In: Wide Field Spectroscopy and the Distant Universe, The $35^{\text {th }}$ Herstmonceux Conference, 3, eds Maddox S. J., Aragon-Salamanca A.

Hamilton A. J. S., 1992. ApJ(Lett), 385, L5.

Hamilton A. J. S., 1998. In: Ringberg Workshop on Large-Scale Structure, TBD, ed. Hamilton D., Kluwer Academic, Dordrecht. 
Hatton S. J., Cole S., 1998. MNRAS, 296, 10.

Heavens A. F., Taylor A. N., 1995. MNRAS, 275, 483.

Hivon E., Bouchet F., Colombi S., Juszkiewicz R., 1995. A\& A, 298, 643.

Jain B., Bertschinger E., 1994. ApJ, 431, 495.

Jensen L. G., Szalay A. S., 1986. ApJ, 305, L5.

Kaiser N., 1984. ApJ(Lett), 284, L9.

Kaiser N., 1987. MNRAS, 227, 1.

Kamionkowski M., Buchalter A., 1998. astro-ph, 9807211.

Kamionkowski M., Spergel D., 1994. ApJ, 432, 7.

Kauffman G., Colberg J. M., Diaferio A., White S. D. M., 1998. astro-ph, 9805283.

Kauffmann G., Nusser A., Steinmetz M., 1997.MNRAS, 286, 795.

Makino N., Sasaki M., Suto Y., 1992. Phys. Rev. D, 46, 585.

Mann R. G., Peacock J. A., Heavens A. F., 1998. MNRAS, 293, 209.

Matarrese S., Verde L., Heavens A., 1997. MNRAS, 290, 651.

Matsubara T., 1995. ApJ Suppl., 101, 1.

Melott A., Shandarin S., 1993. ApJ, 410, 469.

Mo H. J., White S. D. M., 1996. MNRAS, 282, 347.

Peacock J. A., Dodds S. J., 1994. MNRAS, 267, 1020.

Peacock J. A., Heavens A. F., 1985. MNRAS, 217, 805.

Peebles P. J. E., 1980. The Large Scale Structure of the Universe, Princeton University Press.

Pen U.-L., 1997. astro-ph, 9711180.

Politzer H. E., Wise M. B., 1984. ApJ, 285, L1.

Scherrer R. J., Weinberg D. H., 1997. astro-ph, 9712192.

Scoccimarro R., Colombi S., Fry J. N., Frieman J. A., Hivon E., Melott A., 1998. ApJ, 496, 586.

Scoccimarro R., 1997.ApJ, 487, 1.

Sigad Y., Eldar A., Dekel A., Strauss M. A., Yahil A., 1998. ApJ, 495, 516.

Strauss M. A., Willick J. A., 1995. Physics Reports, 261, 271.

Tadros H., Ballinger W. E., Taylor A. N., Heavens A. F., Efstathiou G., Saunders W., Frenk C. S., Keeble O., McMahon R., Maddox S. J., Oliver S., Rowan-Robinson M., Sutherland W. J., White S. D. M., 1998. MNRAS, in preparation.

Taylor A. N., Hamilton A. J. S., 1996. MNRAS, 282, 767.

Tegmark M., Peebles P. J. E., 1998. ApJ(Lett), 500, L79.

Verde L., Heavens A., Matarrese S., Moscardini L., 1998. MNRAS, in press (astro-ph/9806028).

Weinberg D. H., 1995.In: Wide-Field Spectroscopy and the Distant Universe, 129, eds Maddox S. J., Aragon-Salamanca A., Singapore: World Scientific.

\section{APPENDIX A: CORRELATIONS OF HIGH AND LOW REGIONS}

A series expansion for the correlation function of regions above a threshold was obtained by Jensen \& Szalay (1986); see also Politzer \& Wise (1984). The first term in this series is the one obtained by Kaiser (1984), which shows that high regions can be very strongly clustered. In this appendix, we calculate the correlation function of high regions (above a threshold) plus troughs (below a threshold), and show that high regions plus troughs are very weakly correlated on large scales, with a correlation function which is proportional to the square of the correlation function of the field.

We follow the formalism and notation of Jensen \& Szalay (1986). Let the variance in the Gaussian field be $\sigma^{2}$, and consider high regions above $\delta=\nu \sigma$ and troughs below $\delta=-\nu \sigma$.

The two-point correlation function $\xi_{X}$ of regions separated by $r$, with $\nu$ in some region $X$ is given by an integral over the normal bivariate Gaussian:

$1+\xi_{X}=\frac{I^{-2}}{2 \pi \operatorname{det} C} \int_{X} \int_{X} d \nu_{1} d \nu_{2} \exp \left[-\frac{1}{2} \nu_{i} C_{i j}^{-1} \nu_{j}\right]$

where

$I \equiv \int_{X} \frac{d \nu}{\sqrt{2 \pi}} \exp \left(-\nu^{2} / 2\right)$

and the covariance matrix $C_{i j} \equiv\left\langle\nu_{i} \nu_{j}\right\rangle$ is unity on the diagonal, and $\psi=\xi(r) / \sigma^{2}$ off the diagonal.

To evaluate the double integral, we use the same trick as Jensen \& Szalay (1986). We take a Fourier transform of the exponential: 
$\exp \left[-\frac{1}{2}\left(\nu_{i} C_{i j}^{-1} \nu_{j}\right)\right]=\int \frac{d^{2} k}{2 \pi} \exp (-i \mathbf{k} \cdot \nu) \exp \left[-\frac{1}{2}\left(k_{i} C_{i j} k_{j}\right)\right]$.

Then the double integral may be written

$\int_{X} \int_{X} d \nu_{1} d \nu_{2} \int \frac{d^{2} k}{2 \pi} \exp (-i \mathbf{k} \cdot \nu) \exp \left(-k_{i} k_{i} / 2\right) \exp (Q)$

where $Q=-k_{1} k_{2} \psi$. We expand $\exp (Q)=\sum_{m=0}^{\infty} Q^{m} / m$ !, and then regard $Q$ as an operator

$Q \exp (-i \mathbf{k} \cdot \nu)=\psi \frac{\partial}{\partial \nu_{1}} \frac{\partial}{\partial \nu_{2}} \exp (-i \mathbf{k} \cdot \nu)$.

The double integral can then be written

$\int_{X} \int_{X} d \nu_{1} d \nu_{2} \sum_{m=0}^{\infty} \frac{\psi^{m}}{m !}\left(\frac{\partial}{\partial \nu_{1}} \frac{\partial}{\partial \nu_{2}}\right)^{m} \exp \left[-\left(\nu_{1}^{2}+\nu_{2}^{2}\right) / 2\right]$.

Jensen \& Szalay (1986) took the region to be $\nu_{i}>\nu$. We take the region to be $\left|\nu_{i}\right|>\nu$, which gives four contributions to the double integral, and $I$ is also modified to twice the high region result, i.e. $\operatorname{erfc}(\nu / \sqrt{2})$. The terms in the double integral from peak-peak and trough-trough give the same as Jensen \& Szalay (1986), i.e.

$2 \sum_{m=0}^{\infty} \frac{\psi^{m}}{m !}\left[H_{m-1}(\nu) \exp \left(-\nu^{2} / 2\right)\right]^{2}$

where the Hermite polynomial is $H_{m}(x) \equiv \exp \left(x^{2} / 2\right)(-d / d x)^{m} \exp \left(-x^{2} / 2\right)$. The cross-terms in the double integral give an identical contribution, but multiplied by $(-1)^{m}$. The effect of this is to eliminate the odd terms in the series, whilst leaving the even terms intact. The strong clustering term (for high regions alone) which Kaiser computed disappears, leaving the first non-zero contribution as

$\xi_{X}=\frac{\nu^{4}}{2} \psi^{2}+O\left(\psi^{4}\right)$

so if the underlying density field has small correlations on large scales $(\psi \rightarrow 0)$, the field consisting of high regions and low troughs is very weakly clustered. 